\title{
Dip-Pen Nanolithography(DPN): from Micro/Nano-patterns to Biosensing
}

\author{
LI Haonan ${ }^{1,2 \#}$, WANG Zhao ${ }^{1,2 \#}$, HUO Fengwei $^{3}$ and WANG Shutao ${ }^{1,2 \bowtie}$
}

\author{
Received April 30, 2021 \\ Accepted June 6, 2021 \\ (C) Jilin University, The Editorial Depart- \\ ment of Chemical Research in Chinese \\ Universities and Springer-Verlag $\mathrm{GmbH}$
}

$\mathrm{D}$ ip-pen nanolithography is an emerging and attractive surface modification technique that has the capacity to directly and controllably write micro/nano-array patterns on diverse substrates. The superior throughput, resolution, and registration enable DPN an outstanding candidate for biological detection from the molecular level to the cellular level. Herein, we overview the technological evolution of DPN in terms of its advanced derivatives and DPN-enabled versatile sensing patterns featuring multiple compositions and structures for biosensing. Benefitting from uniform, reproducible, and large-area array patterns, DPN-based biosensors have shown high sensitivity, excellent selectivity, and fast response in target analyte detection and specific cellular recognition. We anticipate that DPN-based technologies could offer great potential opportunities to fabricate multiplexed, programmable, and commercial array-based sensing biochips.

Keywords Dip-pen nanolithography; Micro/nano-array pattern; Biosensing

\section{Introduction}

In nature, mammals utilize the olfactory system to perceive scent for keeping themselves safe and healthy. During this process, functional sensorial neuron arrays located in the nose play a crucial role in identifying and discriminating trillions of odorants in various environments. The aligned structure of sensorial neuron arrays significantly simplifies the data collection process and reduces sample consumption, realizing high selectivity and sensitivity, fast response, and real-time monitoring ${ }^{[1,2]}$. Inspired by this unique array-based structure, numerous biosensors with micro/nanoscale arrays are developed for specific biosensing applications ${ }^{[3]}$.

However, it is challenging to fabricate patterned surfaces with controllable shape, size, and chemical composition, especially down to the nanometer scale. Up to date, there are a number of techniques exploited for constructing functional patterned surfaces including photolithography, nanoimprint lithography, electron beam lithography, micro-contact printing, etc. Among these methods, inherent drawbacks have restricted their development for practical sensing applications. For example, photolithography and nanoimprint lithography suffer from finite material selection and low resolution; electron beam lithography is limited by expensive equipment; and micro-contact printing has difficulty in writing multicomponent nanopatterns ${ }^{[4]}$. Therefore, new approaches are required to address these issues.

Building on scanning probe microscope-based lithography, dip-pen nanolithography(DPN) was invented by Mirkin's group in 1999 ${ }^{[5]}$. Since then, DPN has made a great progress in high throughput, diverse inks, and advanced derivative technologies. Micro/nano-array patterns by DPN and its derivatives have enabled a wide range of applications including catalysis ${ }^{[6,7]}$, optics $^{[8]}$, biomedicine ${ }^{[9]}$, and chemical synthesis ${ }^{[10,11]}$. Several reviews have been written devoted to the mechanism of ink transport ${ }^{[12]}$, detailed development of $\mathrm{DPN}^{[13-19]}$, and relevant applications from biomolecular array fabrication $^{[20-22]}$ to material discovery ${ }^{[23-25]}$. In this article, attention is drawn to biosensing based on micro/nano-patterns printed by DPN. Firstly, a brief history of technological development and biosensing applications of DPN is introduced. Then, functional arrays composed of different sensing elements for biological detection via multiple signal read-out approaches are summarized. Target analyte detection using array-based biosensors from molecular level to cellular level is highlighted. Finally, prospects of DPN patterning techniques and DPN-based biosensing are also provided.

The advantages of micro/nanoscale arrays via DPN and its derivatives for biosensing are as follows: (1) high throughput. Different from one single atomic force microscope(AFM) tip, parallel DPN ${ }^{[26]}$ and polymer pen lithography(PPL ${ }^{[27]}$ feature millions of tips to work together for significantly increasing the throughput; (2) high resolution. Conventional DPN has achieved sub-15 $\mathrm{nm}$ resolution with a low throughput ${ }^{[16]}$. Hard-tip, soft-spring lithography(HSL) utilizing a hard Si tip array supported by a spring-like soft layer can reach a sub-50 $\mathrm{nm}$ resolution while simultaneously maintaining a high 

colloidal nanoparticles ${ }^{[43]}$, metal ions ${ }^{[44,45]}$, and sol-gel ${ }^{[46,47]}$. In particular, micro/nano-patterns of biomolecules(proteins, technological development and biosensing applications of DPN and its derivatives are shown in Fig.1. DNA, peptides, lipids, viruses) endow DPN with new

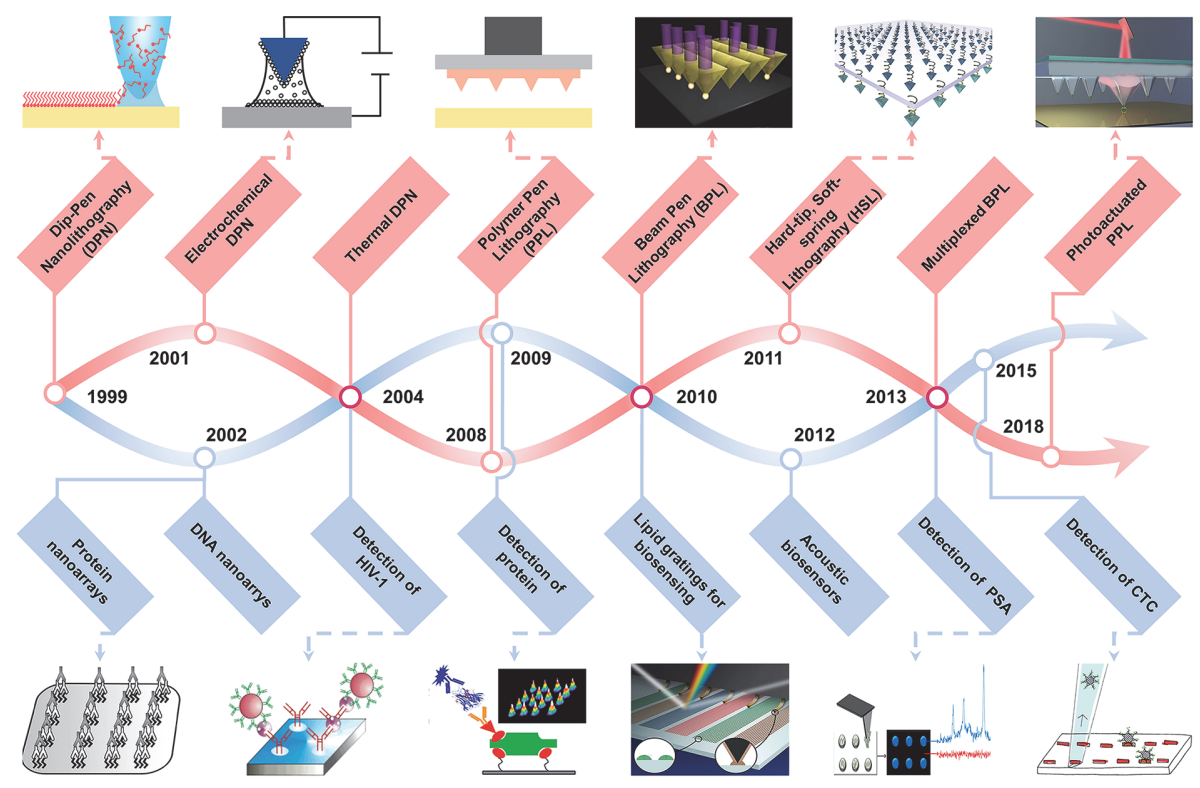

Fig.1 Milestones of technological development and biosensing applications of DPN

The red line shows the evolution from one single tip to parallel array tips and several derivatives of $D P N\left(E-D P N^{[51]}, t P N^{[52]}, P P L^{[27]}, B P L^{[53]}\right.$, HSL ${ }^{[28]}$, and photoactuated PPL that can control single tip in the array $\left.{ }^{[54]}\right)$. The blue one shows several biosensors based on DPN including fabrication of biomolecular arrays (protein ${ }^{[31]}$, DNA ${ }^{[34]}$ and lipid $^{[38]}$ ), biological detection from molecular level(detection of protein ${ }^{[55-57]}$, DNA ${ }^{[58]}$ and virus ${ }^{[59]}$ ) to cellular level(capture of CTC ${ }^{[60]}$ ). Reproduced with permission from ref.[51]. Copyright 2001, American Chemical Society. Reproduced with permission from ref.[53]. Copyright 2010, Springer Nature. Reproduced with permission from ref.[28]. Copyright 2011, Springer Nature. Reproduced with permission from ref.[54]. Copyright 2018, Wiley-VCH. Reproduced with permission from ref.[31]. Copyright 2002, American Association for the Advancement of Science. Reproduced with permission from ref.[59]. Copyright 2004, American Chemical Society. Reproduced with permission from ref.[55]. Copyright 2009, The Royal Society of Chemistry. Reproduced with permission from ref.[56]. Copyright 2010, Springer Nature. Reproduced with permission from ref.[57]. Copyright 2013, American Chemical Society. Reproduced with permission from ref.[60]. Copyright 2015, Springer Nature.

\section{Development of DPN-enabled Biosensing}

In 1999, Piner et al..$^{[5]}$ invented dip-pen nanolithography(DPN) that employed the atomic force microscope(AFM) tip to directly deliver ink molecules to the solid substrate through capillary transport. In this process, the AFM tip acted as a "nib," gold substrate as "paper," and alkanethiols as "ink." The 30-nm molecule-based line was successfully written on a gold thin film in a manner analogous to that of a dip pen ${ }^{[5]}$. However, patterning area and throughput are under restrictions with only one tip working. Since then, many efforts have been made to achieve the mode of multi-pen writing to increase the throughput. In 2000, Hong et al. ${ }^{[26]}$ firstly reported parallel DPN with one-dimensional(1D) multi-pen cantilever arrays. Only one tip in the array had a feedback system to monitor the printing process, while the other tips performed passive parallel writing. Accordingly, massively parallel 2D DPN was developed in 2006. The throughput of DPN was dramatically improved utilizing a 55000-pen 2D array fabricated by photolithographic techniques ${ }^{[61]}$. Since this initial demonstration, the tip number of 2D parallel DPN has been scaled to as many as 1.3 million despite high-expense and fragility ${ }^{[13]}$.

A significant breakthrough is realized by introducing the creative concept of cantilever-free scanning probe lithography. In 2008, Huo et al. ${ }^{[27]}$ first reported polymer pen lithography (PPL) that used a soft elastomeric polydimethylsiloxane (PDMS) tip array to deliver 16-mercaptohexadecanoic acid (MHA) onto gold substrates. With more than 11 million pyramid-shaped pens, PPL can construct arbitrary complex patterns in a rapid, high-throughput, and large-area manner. To improve the resolution, hard-tip, soft-spring lithography (HSL) was demonstrated in 2011. Soft PDMS tips were replaced by hard silicon tips with a diameter of $22 \mathrm{~nm}$, which enabled HSL to pattern nanoscale features smaller than $50 \mathrm{~nm}$ over centimeter-scale areas ${ }^{[28]}$.

Apart from the direct delivery of molecules, DPN has been 
used to directly deliver different kinds of energy including electrical, thermal, mechanical, and optical energy. The idea of electrochemical dip-pen nanolithography(E-DPN) was first demonstrated by Liu's group ${ }^{[51]}$ in 2001. The water meniscus between the tip and substrate worked as a tiny electrochemical cell. Metal ions could be dissolved, reduced electrochemically, and deposited on the surface to construct metal and semiconductor features with nanometer dimensions ${ }^{[51]}$. In 2004 Sheehan et al. ${ }^{[52]}$ utilized a heated AFM tip to melt and deposit solid organic inks on the surface. One impressive advantage of tDPN is to use solid inks to build up multilayer multicomponent patterns, thus allowing for 3D nanostructures. Dip-pen nanodisplacement lithography(DNL), a DPN derivative with the input of mechanical energy, was exploited to manipulate 2D and 3D nanostructures of polymer brushes. The self-assembled monolayer(SAM) molecules were cleaved away mechanically and replaced by the initiator of surfaceinitiated atom transfer radical polymerization(SI-ATRP). By regulating the DNL printing parameters, one can obtain combinative libraries of chemical structure, topography, size, shape, grafting density, and chain configuration of polymer brushes ${ }^{[62]}$. Light can also be directly delivered to the surface by beam pen lithography(BPL). Based on PPL, the apex of PDMS tips in the array was removed to create apertures to allow UV-light passing. Superior to traditional photolithography, maskless BPL can pattern feature dots below the diffraction limit of the light source due to the nearfield effect between the tip and surface ${ }^{[53]}$.

DPN can precisely control the patterning process at the nanometer-size scale and produce highly identical array patterns due to simultaneous motions of the tip array. In Fig.2, array patterns consisting of basic constitutive elements including dot, ring, rectangle, line, Y-shape, and multielement are summarized. When used for biosensing applications, array-based structures are beneficial for the highly sensitive detection process because the total area occupied by the fixed number of targets of array-based sensors can be dramatically reduced. This translates into vastly decreased sample volumes and allows for detecting a smaller amount of target molecules for a given analyte concentration, leading to lower limits of detection. DPN with its derivatives is also capable of writing arbitrary patterns over square centimeter areas. In 2008, 15000 replicas of the 2008 Beijing Olympic logo were printed on gold with MHA as the ink and subsequent wet chemical etching ${ }^{[27]}$. Based on beam pen lithography(BPL), multiplexed BPL was developed by incorporating a digital micromirror device (DMD) system. The light source can be selectively directed onto the back of a near-field aperture array to induce local photochemical reaction creating arbitrary patterns. Functional electronic devices including inductors, capacitors, and surface acoustic wave (SAW) sensors by multiplexed BPL performed normal functions, indicating successful patterning of active circuits. Besides, a world map over centimeter-scale areas was also fabricated in a standard lift-off process ${ }^{[48]}$. In 2013, a heated tip was used to control the density of amine groups on the polymer film by the applied temperature and scanning speed. The programmed gradient patterns of the Mona Lisa were fluorescently labeled and subsequently imaged with fluorescence microscopy ${ }^{[49]}$.

The rapid technological progress of DPN provides more possibilities to fabricate biomolecular arrays for biosensing applications. In 2002, Lee et al. ${ }^{[31]}$ constructed nanoscale protein arrays by DPN in an indirect strategy. MHA feature dots were printed and the areas surrounding MHA were passivated. Proteins could be absorbed in preformed MHA patterns via electrostatic interactions ${ }^{[31]}$. Demers et al. ${ }^{[34]}$ utilized direct-write DPN to generate nanoscale DNA patterns on both metallic and insulating substrates. Modification of DNA with hexanethiol

(A) Micro/nanoscale arrays

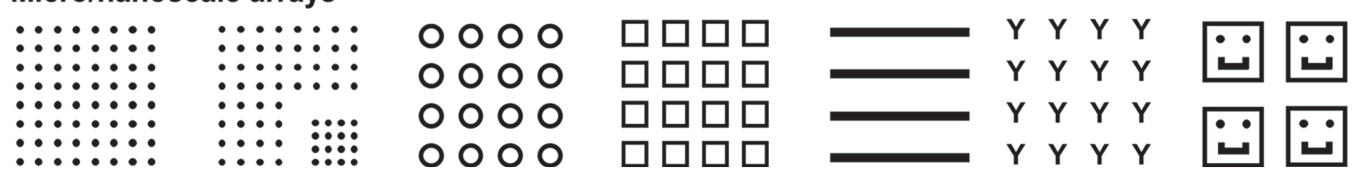

Arbitrary patterns
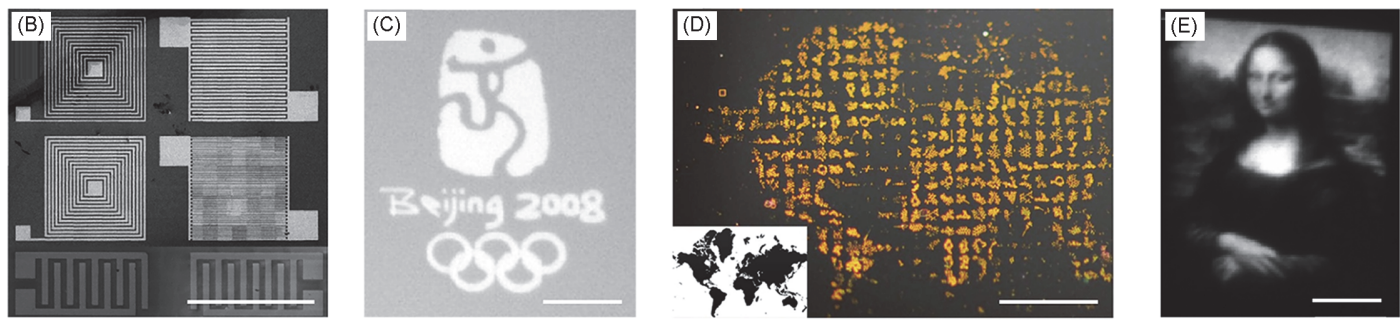

Fig.2 Different arrays and patterns fabricated by DPN and its derivative technologies including micro/nanoscale arrays composed of basic constitutive elements(dot, ring, rectangle, line, Y-shape, and multielement, A) and arbitrary patterns[active circuits $^{[48]}(B)$, the 2008 Beijing Olympic $\log _{o^{[27]}}(C)$, the world map ${ }^{[48]}(D)$, and the Mona Lisa $\left.{ }^{[49]}(E)\right]$

Scale bar: (B) $1 \mathrm{~mm}$; (C) $20 \mu \mathrm{m}$; (D) $2 \mathrm{~mm}$; (E) $10 \mu \mathrm{m}$. Reproduced with permission from ref.[48]. Copyright 2013, Springer Nature. Reproduced with permission from ref.[27]. Copyright 2008, American Association for the Advancement of Science. Reproduced with permission from ref.[49]. Copyright 2013, American Chemical Society. 


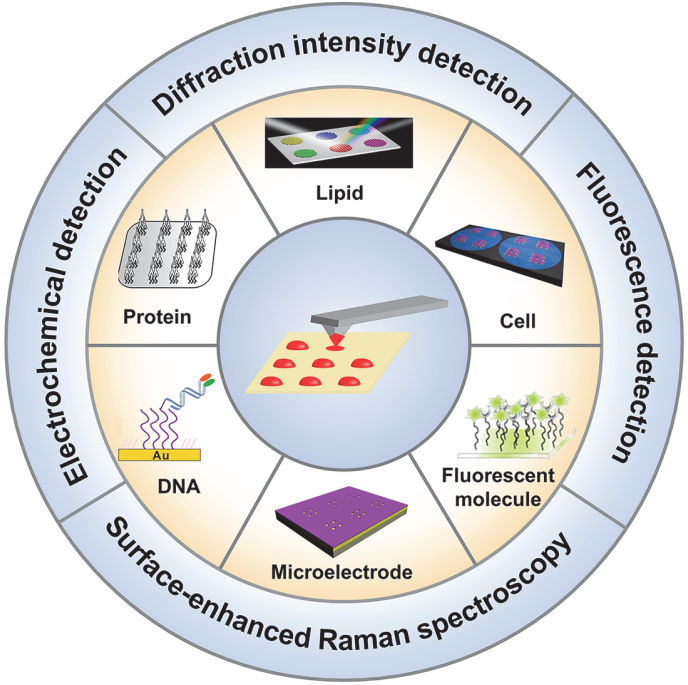

Fig.3 Recent research concerning DPN-enabled micro/nanopatterns based on different sensing elements with relevant signal read-out ways

By taking advantaging of protein ${ }^{[31]}$, DNA ${ }^{[63]}$, lipid $^{[64]}$, fluorescent molecule $\mathrm{e}^{[65,66]}$, microelectrode ${ }^{[67]}$ and cell-based arrays ${ }^{[68]}$, highly sensitive and selective biosensors are developed with fluorescence, surface-enhanced Raman spectrascopy(SERS), electrochemistry, and diffraction intensity read-out approaches. Reproduced with permission from ref.[31]. Copyright 2002, American Association for the Advancement of Science. Reproduced with permission from ref.[63]. Copyright 2014, American Chemical Society. Reproduced with permission from ref.[65]. Copyright 2008, Wiley-VCH. Reproduced with permission from ref.[67]. Copyright 2017, The Royal Society of Chemistry. Reproduced with permission from ref.[68]. Copyright 2017, American Chemical Society.

groups allowed patterning on gold, and oligonucleotides containing 5'-terminal acrylamide groups could be written on derivatized silica ${ }^{[34]}$. Nanoarrays of protein and DNA opened up new opportunities for biosensors based on surfacemediated biological recognition processes. Besides, biosensors of lipid, fluorescent molecules, and $\mathrm{Au}$ microelectrodes are also fabricated for detecting various target analytes with different read-out approaches[fluorescence, surface-enhanced Raman spectrascopy(SERS), electrochemis-try, diffraction density, etc.](Fig.3). The detailed demonstra-tion of biosensors based on micro/nano-array patterns by DPN will be discussed in the following part.

\section{Biosensing Applications Based on DPN- enabled Patterns}

\subsection{Molecule Detection}

DPN has offered new functionalities and possibilities towards a wide variety of emerging biosensing applications. Table 1 detailedly summarizes biological detection of micro/nanopatterns by DPN in terms of different detection targets, detection elements, and detection approaches.

\subsubsection{Detection of Protein}

Protein plays a unique part in the biological process and the detection of protein is of great significance for the diagnosis of diseases. Immunoassays based on antigen-antibody interactions are widely used to detect protein biomarkers. In 2009, Lee et al. ${ }^{[55]}$ developed an ultra-sensitive nanoarray chip consisting of a single-molecule sandwich immunoassay for

Table 1 Biosensing applications of micro/nano-patterns by DPN*

\begin{tabular}{|c|c|c|c|c|}
\hline Molecule & Detection target & Detection element & Detection approach & Ref. \\
\hline \multirow[t]{10}{*}{ Protein } & Biotin-streptavidin interaction & Superparamagnetic beads & Magnetic detection & [69] \\
\hline & Protein & Sandwich immunoassay & Fluorescence detection & {$[55,70]$} \\
\hline & ERK2-KSR interaction & ERK2-KSR binding microarray & Fluorescence detection & [71] \\
\hline & PSA & Sandwich immunoassay & Resonance Raman scattering detection & [57] \\
\hline & Serum proteins & scFv antibody array & Fluorescence detection & {$[72,73]$} \\
\hline & Proteins & Lipid gratings & Diffraction intensity detection & [56] \\
\hline & Proteins & $\mu \mathrm{F}$-on-SAW device & Acoustic detection & [74] \\
\hline & Proteins & Lipid gratings on graphene & Fluorescence detection & {$[75,76]$} \\
\hline & Proteins & Lipid functionalized microresonators & Laser detection & [77] \\
\hline & Sar1 protein-lipid interaction & Lipid gratings & Diffraction intensity detection & [78] \\
\hline \multirow[t]{5}{*}{ DNA } & DNA Hybridization & ssDNA & Topographic changes by AFM & [58] \\
\hline & DNA & DNA-AuNPs & Electrochemical detection & [79] \\
\hline & DNA & Plasmonic gold surface & SERS detection & {$[80]$} \\
\hline & DNA & DNA-AuNPs & Colorimetric detection & {$[81,82]$} \\
\hline & DNA & Gold nanodisc arrays & LSPR spectroscopy & [83] \\
\hline \multirow[t]{4}{*}{ Other analytes } & $\mathrm{pH}$ & Fluorescein dot arrays & Fluorescence detection & {$[66]$} \\
\hline & $\mathrm{Cu}^{2+}, \mathrm{Ca}^{2+}$ & Fluorescent SAM & Fluorescence detection & {$[65]$} \\
\hline & $\mathrm{H}_{2} \mathrm{O}_{2}$ & Au microelectrode arrays & Electrochemical detection & [67] \\
\hline & HIV-1 & Sandwich immunoassay & Topographic changes by AFM & [59] \\
\hline
\end{tabular}

* ssDNA: single-stranded DNA, AFM: atomic force microscope, DNA-AuNPs: DNA-Au nanoparticles, SERS: surface-enhanced Raman scattering, LSPR: localized surface plasmon resonance, ERK: extracellular regulated protein kinases, KSR: kinase suppressor of ras, PSA: prostate-specific antigen, svFc: single-chain fragment variable, $\mu$ F-on-SAW: microfluidics-on-Surface Acoustic Wave, HIV-1: human immunodeficiency virus type 1, SAR: Secretion-associated and Ras-related, SAM: self-assembled monolayer. 
protein detection in biologic fluids. Maleimide- $\mathrm{PEO}_{2}$-biotin was coated on AFM tip and was subsequently written on (3-mercapto-propyl)trimethoxysilane(MPTMS)-coated glass surfaces. Biotinylated protein G, which specifically binds to IgG and is available for capturing antigen, was used for antibody 1 immobilization. Through total internal reflection fluorescence microscopy(TIRFM), the structure of singlemolecule sandwich antibody 1 -antigen-antibody $\mathbf{2}$ was found to increase target protein quantification to the $\mathrm{zM}$-aM level $\left(\times 10^{-21}-10^{-18} \mathrm{~mol} / \mathrm{L}\right)$. Two kinds of cytokines(TNF-a and IL-1a) in pre-pared media and clinical samples were clearly differen-tiated by TIRFM ${ }^{[55]}$. Similarly, a sandwich immunoassay for prostate-specific antigen(PSA) was fabricated by DPN and the signal of resonance Raman was detected. The detection limit achieved was lower than those obtained with similar assay formats of fluorescence detection $^{[57]}$. As illustrated in Fig.4, Petersson and co-workers ${ }^{[72}$ exploited a miniaturized planar antibody array for serum protein profiling targeting biomarkers in systemic lupus erythematosus(SLE) nephritis. Human recombinant singlechain fragment variable(scFv) antibodies were directly printed in $10 \mu \mathrm{m}$-sized spots. After blocked and washed, the biochip was incubated with biotinylated serum samples to capture antibody-specific protein. Finally, streptavidin with fluorescent molecules was immobilized. The multiplexed array of 48 antibodies interfaced with a high-resolution fluorescent-based scanner enabled fewer antibodies and samples consumed and more analytes detected [73].

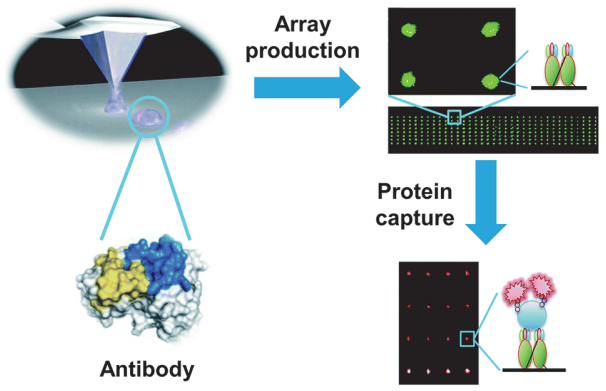

Fig.4 Schematic of the miniaturized 48-plex recombinant antibody array platform for protein detection

Reproduced with permission from ref.[73]. Copyright 2014, The Royal Society of Chemistry.

Besides immunoassays for protein detection, lipid multilayer gratings are also explored to achieve biosensing. Optical diffraction gratings of biofunctional lipid multilayers were generated to realize label-free and specific detection of lipid-protein interactions in solution. When analytes bound to phospholipids with functional headgroups, diffraction density was recorded in response to the change of phospholipid superstructures ${ }^{[56]}$. Furthermore, this functional lipid platform was combined with acoustic biosensors ${ }^{[74]}$, microresonators ${ }^{[77]}$, and graphene ${ }^{[75,76]}$ for further biological detection in different forms of signal read-out.

\subsubsection{Detection of DNA}

DNA detection has intensive applications concerning genetics, pathology, criminology, and food safety. In 2003, Zhou et al. ${ }^{[58]}$ used AFM as a tool to quantitatively detect label-free DNA hybridization and relevant dynamics by measuring height changes of DNA nanofeatures. Electrochemical approaches are also available for DNA detection. For example, a DNA chip, which had $5 \mu \mathrm{m} \times 10 \mu \mathrm{m}$ electrical gaps for patterning capture ssDNAs, was developed in 2008. Following successful hybridization of capture-target DNA, gold nanoparticle functionalized ssDNA bound to the target DNA with an evident drop of resistance across the gap. In this method, the lowest detection limit could reach $10 \mathrm{pmol} / \mathrm{L}^{[79]}$. By colorimetric detection, as little as $30 \mathrm{ng}$ of Ganoderma-derived DNA could be detected without any polymerase chain reaction(PCR) amplification. As shown in Fig.5, reporter DNA arrays were written on Au substrates by PPL. After passivation of unpatterned region and hybridization of target DNA, gold nanoparticles were modified with target DNA. By naked eyes, one can visually observe the result in the presence of the target DNA $^{[82]}$

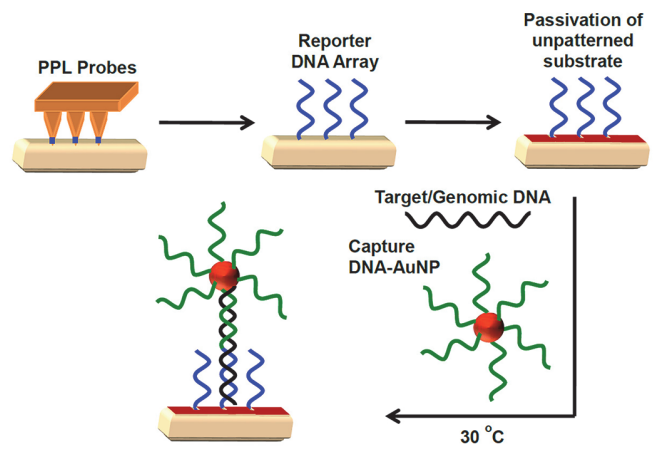

Fig.5 Schematic diagram showing the immobilization of reporter DNA using PPL on Au surface followed by the passivation of the unpatterned substrate

In the presence of capture DNA-AuNPs, the target DNA forms a sandwich assay that can be visualized optically.

\subsubsection{Detection of Other Analytes}

Besides protein and DNA, functional patterned surfaces can also be applied to detect other target analytes. Human immunodeficiency virus type $1(\mathrm{HIV}-1)$ in plasma obtained directly from HIV-1-infected patients was detected by nanoscale patterns of antibodies generated via DPN. To this end, anti-p24 antibodies were immobilized on the patterned MHA dot features followed by immersing in a plasma sample containing HIV-1 p24 antigen. Gold nanoparticle probes, 
functionalized with polyclonal antibodies to the p24 antigen, were reacted with the nanoarrays to amplify the signal. Such assays could dramatically decrease the detection limit compared with conventional enzyme-linked immunosorbent assay(ELISA)-based immunoassays ${ }^{[59]}$.

The sensitive measurement of $\mathrm{H}_{2} \mathrm{O}_{2}$ is of great importance both in biological studies and electrochemical biosensors. In 2017, size-tunable, highly sensitive microelectrode arrays (MEAs) were developed to detect $\mathrm{H}_{2} \mathrm{O}_{2}$ electrochemically [Fig.6(A)]. MHA feature dots were written on gold substrates by PPL. Polymethyl methacrylate(PMMA) brushes were grown, where the initiator was backfilled through surfaceinitiated atom transfer radical polymerization(SI-ATRP). Finally, Prussian blue with extraordinary electrocatalytic properties was electrochemically deposited onto the $\mathrm{Au}$ microelectrode arrays. The detection limit could be as low as $5 \mathrm{nmol} / \mathrm{L}$ in such an illustration ${ }^{[6]]}$.

Fluorescent molecule arrays are generally used for biological detection by monitoring the changes in fluorescence intensity. Basabe-Desmonts et al. ${ }^{[65]}$ transferred metal ions onto the fluorescent SAM-functionalized glass substrates by DPN. Two ions had different responses to the fluorescent surfaces: $\mathrm{Ca}^{2+}$ was complexed on the organic monolayer, leading to an enhancement of fluorescent signal, whereas complexation of $\mathrm{Cu}^{2+}$ ions resulted in quenching of fluorescence emission. Thus, metal ions and fluorescent patterns were simultaneously generated on the substrates ${ }^{[65]}$.

A pH-responsive fluorescent nanoarray by direct-write parallel DPN was showed in Fig.6(B). Fluorescein was chosen as the proof-of-concept candidate because it has different $\mathrm{pH}$-dependent ionization forms with distinct optical properties. Fluorescein arrays exhibited an increasing fluorescence signal when exposed to a basic atmosphere, which could be attributed to the conversion of the monoanionic form into the dianionic state. However, due to the protonation of fluorescein to produce a neutral, nonfluorescent form, the fluorescence signal was abruptly decreased in an acid atmosphere ${ }^{[66]}$.
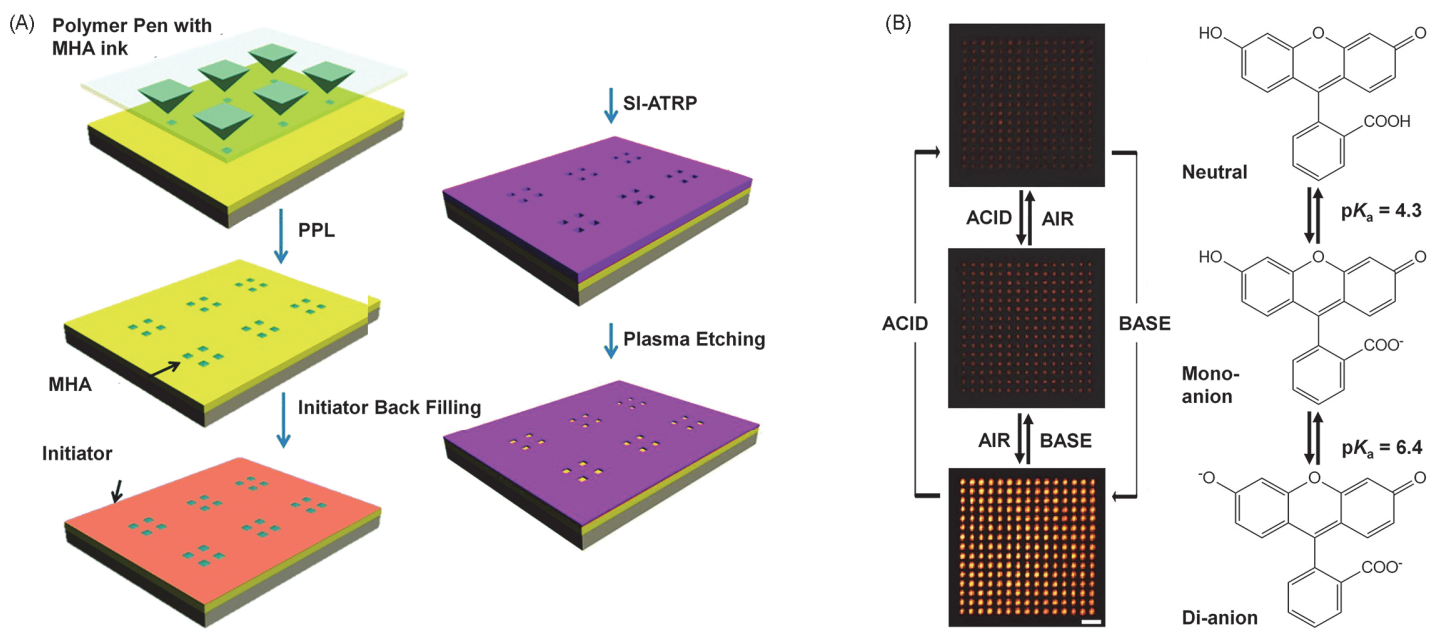

Fig.6 Schematic fabrication of the MEAs by PPL for $\mathrm{H}_{2} \mathrm{O}_{2}$ detection electrochemically(A), confocal fluorescence microscopy images of the array of fluorescein dots showing the fluorescence emission changes after exposure to ambient conditions (middle), acidic conditions(top), and basic conditions(bottom)(B)

(B) Chemical structures corresponding to the predominant fluorescein form at each pH range. (A) Reproduced with permission from ref.[67]. Copyright 2017, The Royal Society of Chemistry. (B) Reproduced with permission from ref.[66]. Copyright 2008, Wiley-VCH.

\subsection{Cell Recognition}

\subsubsection{Cell-based Assays}

DPN, capable of patterning multiple materials, can fabricate suitable microenvironments wherein a single cell can be isolated, cultured, and analyzed. Profiling of allergic responses is of significant interest for fundamental researches of allergology and diagnosis of allergic diseases. Recently, lipid dip-pen nanolithography(L-DPN) was utilized for a series of mast cell sensitization investigations through allergen arrays comprising of dinitrophenol(DNP) ligand mixed with functional phospholipids. A model system was established to detect allergen-specific Immunoglobin $\mathrm{E}(\mathrm{IgE})$ antibodies by analyzing the response of sensitized, fluorescently labeled mast cells on the allergen patterns. Co-localization of labeled IgE receptors on the allergen features could be observed as soon as the mast cells adhered to the patterned glass surface, which was considered as IgE-FceRI clusters ${ }^{[84]}$. Furthermore, the glucocorticoid receptor tagged with green fluorescent protein was recruited to the plasma membrane of mast cells on the allergen arrays, which could be visualized by total internal reflection(TIRF) microscopy ${ }^{[85]}$. Patterned surfaces of allergen arrays were also incorporated into a microfluidic chip for mast cell activation studies and cell-sorting applications. By 
fluorescence microscopy, cell suspension, incubation process, and read-out for cell activation screening could be easily adapted in a microfluidic chip. The platform of allergen arrays, where the allergen had pronounced retention of bone marrowderived mast cells(BMMCs), was employed to identify sensitized BMMCs in a background of either unsensitized BMMCs or adherent cells ${ }^{[86]}$.

Cellular enzymatic activities were also assessed via self-assembled monolayer laser desorption-ionization(SAMDI) mass spectrometry employing PPL to guide cell localization.
As shown in Fig.7, HeLa cells were first attached to the individual nanoarrays, then were cultured, and treated with small molecules. After the medium was removed and the cells were lysed, phosphatase enzymes from cells converted immobilized phosphopeptides on the substrate to the dephosphorylated form. SAMDI spectra reported a corresponding peak appearing at 80 Da lower mass, which was consistent with the dephosphorylation of phosphopeptides so that the information about the amount of enzyme activity in the cell could be obtained ${ }^{[68]}$.
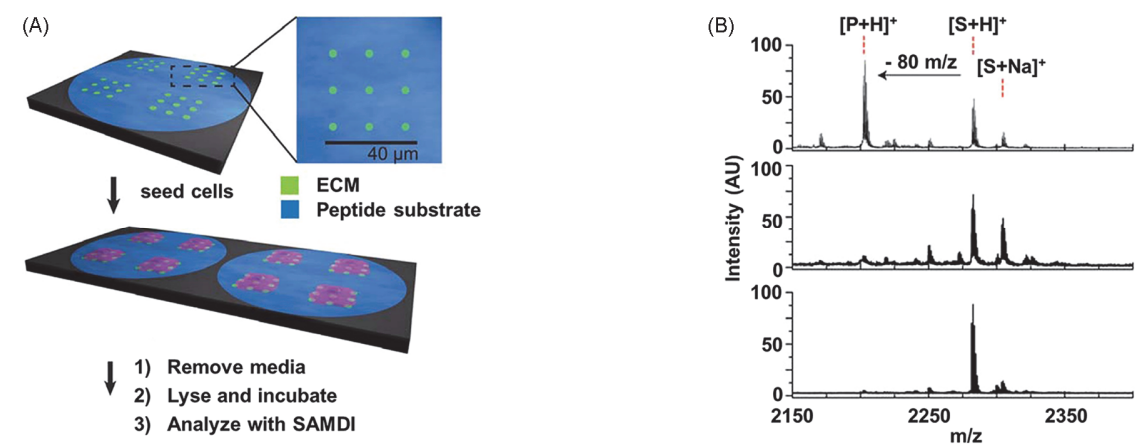

Fig.7 Schematic of cell-based assays where patterned regions are written with extracellular matrix proteins for cell adhesion and unpatterned regions are modified with phosphopeptide(A) and SAMDI spectra of the surface after removal of the lysate(B) Reproduced with permission from ref.[68]. Copyright 2017, American Chemical Society.

\subsubsection{Capture of CTC}

Efficient capture, isolation, and subsequent analysis of circulating tumor cells(CTC) in the diagnosis of early diseases are highly desirable. Many biointerfaces are fabricated to meet the demand for sensitive, selective, portable, and low-cost detection of CTC ${ }^{[87-92]}$. As shown in Fig.8, In 2015, Brinkmann et al. ${ }^{[60]}$ printed micropatterns of biotin-4-fluorescein on bovine serum albumin(BSA)-coated glass surfaces by PPL. The combination of micropatterns functionalized with streptavidin and a microfluidic chaotic mixing system enabled target cells immobilized on the microarray after incubation with an antibody cocktail. It was also found that the microarray platform was allowed for subsequent recovery of target cells for further analysis. By analyzing blood samples from cancer patients, the platform also demonstrated great feasibility for clinical applications ${ }^{[60]}$.

In 2020, Liu et al. ${ }^{[93]}$ utilized PPL to print reactive binding sites for detecting CTCs. By exploring the impact of staggered herringbone structure of microfluidic biochip and target cell concentration on the capture efficiency of CTC capture, they got an optimized protocol of CTC capture platform. Furthermore, the viability of captured tumor cells, as well as single-cell recovery for downstream genomic analysis was also demonstrated.
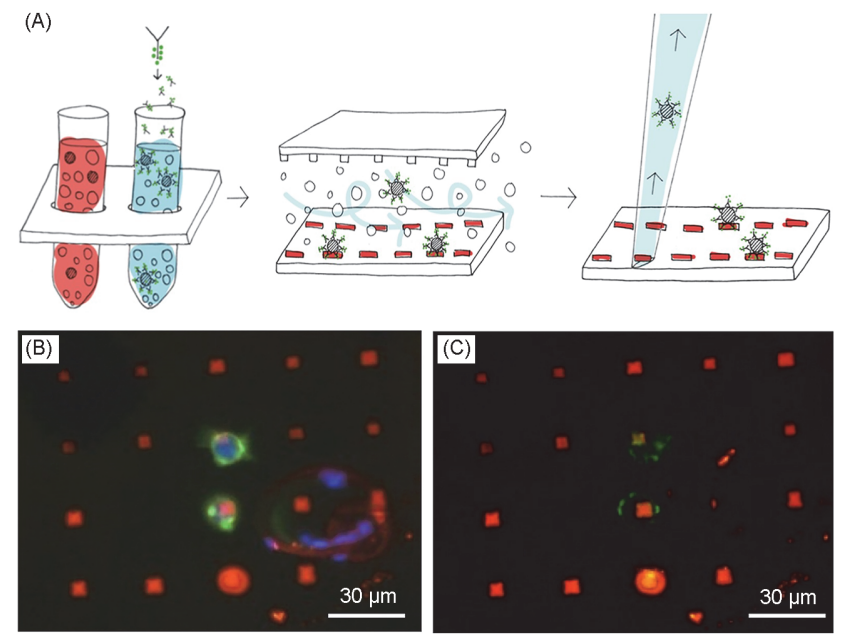

Fig. 8 CTC capture and extraction based on the micropattern platform(A), the micrograph illustrates an overlay of the Texas Red, FITC, and DAPI channels showing the streptavidinpattern in red, the cell nucleus in blue, and anti-EPCAM in green of two co-localized MCF-7 cells(B), after removal of the cell by micromanipulation, little residues of anti-EpCAM remain on the surface(C)

Reproduced with permission from ref.[58]. Copyright 2015, Springer Nature.

\section{Summary and Outlook}

DPN has been rapidly developed over the past decades owing to its high resolution, high throughput, high registration, large scale printing, diverse material selection, and low cost over other nanofabrication techniques. The inks and substrates 

mechanisms of cancer propagation, wound repair, and mode. Especially, PPL merges size control with large-area printing merits to provide an excellent model for BPL and HSL. Besides, the derivatives with external inputs have also been developed, including E-DPN, tDPN, and DNL. The advances of DPN surface fabrication technology pave the way for fundamental research and practical biosensing applications Successful fabrication of highly specific biological arrays presents a cornerstone for highly sensitive biodetection and accurate bioanalysis. Although great achievements have been made, there are still some challenges to be addressed for biosensors based on DPN-enabled array patterns.

\subsection{Multicomponent Arrays for Multiplexed Biosen- sors}

Multiplexing biosensors, aimed to detect several analytes simultaneously, are of immense scientific interest for accurately diagnosing and treating a specific disease. Although there are several strategies for creating multicolor patterns by DPN and its derivatives ${ }^{[94-97]}$, current pattern complexity is hindered by the limitations imposed by the passive probe design. Therefore, it is of paramount interest to understand different inks' transportation mechanisms and relevant post-functionalization to realize multicomponent arrays for multiplexed detection.

\subsection{CRISPR-mediated Biosensing}

Clustered regularly interspaced short palindromic repeats(CRISPR) is the most used and well-known genome engineering tool. CRISPR-based biosensing has the potential to finish the dual task for analyte recognition and signal transduction in a programmable and selective mechanism. DPN can be integrated with the CRISPR molecular system to fabricate multifunctional nanoarrays for biomolecular sensors, holding promise as a valuable platform for understanding cellular pathways, detecting environmental pathogens, and diagnosing early diseases.

\subsection{Functional 3D Micro/nano-structures}

As a surface patterning technique, it is difficult for DPN to build 3D structures. Although there are some efforts made to fulfill additive manufacturing ${ }^{[98,99]}$, a true 3D DPN that directly delivers inks onto the substrates in a high-resolution, highthroughput, and large-area manner to create 3D structures should be developed. 3D DPN will extend the scope of 3D printing down to nanometer scales. It is particularly attractive for advanced 3D micro/nano-structures to mimic the real

\subsection{Biochips for Virus Detection}

In 2019, severe acute respiratory syndrome coronavirus 2(SARS-CoV-2) has caused an abrupt outbreak of coronavirus disease 2019(COVID-19). Public healthcare systems and the safety of human lives all over the world are threatened severely. Current detection methods require expensive equipment, professional operation, and long turn-around time from sample to result ${ }^{[100]}$. It is meaningful and necessary to develop simple, fast, and accurate detection biochips that can be used in schools, families, and remote areas. It is high time for DPN-based biosensors to go out of the lab to detect infectious viruses massively. We believe that uniform, largescale, and nanoscale biomolecular arrays generated by DPN will make a significant difference for next-generation biochip manufacture.

From the first Chinese writing brush to the invention of printing, it takes mankind over one thousand years. When it comes to molecular printing, the evolution from one tip of DPN to millions of tips of PPL only costs less than 10 years. We believe that through DPN, one can create molecular patterns as wanted in a reproducible, controllable, easy-use, and universal fashion after simple training in the future. On this basis of advanced technology, array-based biosensors for biodetection and bioanalysis will also make a significant contribution to people's lives and health.

\section{Conflicts of Interest}

The authors declare no conflicts of interest.

\section{References}

[1] Shepherd G. M., Nature, 2006, 444, 316

[2] Guerrini L., Garcia-Rico E., Pazos-Perez N., Alvarez-Puebla R. A., ACS Nano, 2017, 11, 5271

[3] Geng Y., Peveler W. J., Rotello V. M., Angew. Chem. Int. Ed., 2019, 58, 5190

[4] Nie Z., Kumacheva E., Nat. Mater., 2008, 7, 277

[5] Piner R. D., Zhu J., Xu F., Hong S. H., Mirkin C. A., Science, 1999, 283, 661

[6] Huang L., Chen P. C., Liu M., Fu X., Gordiichuk P., Yu Y., Wolverton C., Kang Y., Mirkin C. A., Proc. Natl. Acad. Sci. USA, 2018, 115, 3764

[7] Kluender E. J., Hedrick J. L., Brown K. A., Rao R., Meckes B., Du J. S., Moreau L. M., Maruyama B., Mirkin C. A., Proc. Natl. Acad. Sci. USA, 2019, 116,40

[8] Du J. S., Shin D., Stanev T. K., Musumeci C., Xie Z., Huang Z., Lai M., Sun L., Zhou W., Stern N. P., Dravid V. P., Mirkin C. A., Sci. Adv., 2020, 6, eabc4959

[9] Cabezas M. D., Meckes B., Mirkin C. A., Mrksich M., ACS Nano, 2019, 13, 11144

[10] Chen P. C., Liu M., Du J. S., Meckes B., Wang S., Lin H., Dravid V. P. Wolverton C., Mirkin C. A., Science, 2019, 363, 959

[11] Huang L., Lin H., Zheng C. Y., Kluender E. J., Golnabi R., Shen B., Mirkin C. A., J. Am. Chem. Soc., 2020, 142, 4570

[12] Brown K. A., Eichelsdoerfer D. J., Liao X., He S., Mirkin C. A., Front. Phys., 2014, 9,385 
[13] Braunschweig A. B., Huo F., Mirkin C. A., Nat. Chem., 2009, 1, 353

[14] Liu G., Hirtz M., Fuchs H., Zheng Z., Small, 2019, 15, 1900564

[15] Garcia R., Knoll A. W., Riedo E., Nat. Nanotechnol., 2014, 9, 577

[16] He Q., Tan C., Zhang H., ACS Nano, 2017, 11, 4381

[17] Brown K. A., Hedrick J. L., Eichelsdoerfer D. J., Mirkin C. A., ACS Nano, 2019, 13,8

[18] Giam L. R., Mirkin C. A., Angew. Chem. Int. Ed., 2011, 50, 7482

[19] Ginger D. S., Zhang H., Mirkin C. A., Angew. Chem. Int. Ed., 2004, 43, 30

[20] Wu C. C., Reinhoudt D. N., Otto C., Subramaniam V., Velders A. H., Small, 2011, 7, 989

[21] Liu X., Carbonell C., Braunschweig A. B., Chem. Soc. Rev., 2016, 45, 6289

[22] Carbonell C., Braunschweig A. B., Acc. Chem. Res., 2017, 50, 190

[23] Salaita K., Wang Y., Mirkin C. A., Nat. Nanotechnol., 2007, 2, 145

[24] Liu G., Petrosko S. H., Zheng Z., Mirkin C. A., Chem. Rev., 2020, 120, 6009

[25] Li Z.-Q., Guo P., Zhou Y.-G., Adv. Mater. Technol., 2021, 2000897

[26] Hong S. H., Mirkin C. A., Science, 2000, 288, 1808

[27] Huo F. W., Zheng Z. J., Zheng G. F., Giam L. R., Zhang H., Mirkin C. A Science, 2008, 321, 1658

[28] Shim W., Braunschweig A. B., Liao X., Chai J. N., Lim J. K., Zheng G. F. Mirkin C. A., Nature, 2011, 469, 516

[29] Lim J. H., Mirkin C. A., Adv. Mater., 2002, 14, 1474

[30] Noy A., Miller A. E., Klare J. E., Weeks B. L., Woods B. W., DeYoreo J. J., Nano Lett., 2002, 2, 109

[31] Lee K. B., Park S. J., Mirkin C. A., Smith J. C., Mrksich M., Science, 2002, 295, 1702

[32] Lee K. B., Lim J. H., Mirkin C. A., J. Am. Chem. Soc., 2003, 125, 5588

[33] Lim J. H., Ginger D. S., Lee K. B., Heo J., Nam J. M., Mirkin C. A., Angew. Chem. Int. Ed., 2003, 42, 2309

[34] Demers L. M., Ginger D. S., Park S. J., Li Z., Chung S. W., Mirkin C. A. Science, 2002, 296, 1836

[35] Wilson D. L., Martin R., Hong S., Cronin-Golomb M., Mirkin C. A., Kaplan D. L., Proc. Natl. Acad. Sci. USA, 2001, 98, 13660

[36] Cho Y., Ivanisevic A., J. Phys. Chem. B, 2004, 108, 15223

[37] Jiang H. Z., Stupp S. I., Langmuir, 2005, 21, 5242

[38] Lenhert S., Sun P., Wang Y., Fuchs H., Mirkin C. A., Small, 2007, 3, 71

[39] Bian S., He J., Schesing K. B., Braunschweig A. B., Small, 2012, 8, 2000

[40] Cheung C. L., Camarero J. A., Woods B. W., Lin T. W., Johnson J. E., De Yoreo J. J., J. Am. Chem. Soc., 2003, 125, 6848

[41] Smith J. C., Lee K. B., Wang Q., Finn M. G., Johnson J. E., Mrksich M., Mirkin C. A., Nano Lett., 2003, 3, 883

[42] Rozhok S., Shen C. K. F., Littler P. L. H., Fan Z. F., Liu C., Mirkin C. A., Holz R. C., Small, 2005, 1, 445

[43] Gundiah G., John N. S., Thomas P. J., Kulkarni G. U., Rao C. N. R., Heun S. Appl. Phys. Lett., 2004, 84, 5341

[44] Li J. Y., Lu C. G., Maynor B., Huang S. M., Liu J., Chem. Mater., 2004, 16 , 1633

[45] Ding L., Li Y., Chu H. B., Li X. M., Liu J., J. Phys. Chem. B, 2005, 109, 2233

[46] Su M., Liu X. G., Li S. Y., Dravid V. P., Mirkin C. A., J. Am. Chem. Soc., 2002 124,1560

[47] Fu L., Liu X. G., Zhang Y., Dravid V. P., Mirkin C. A., Nano Lett., 2003, 3, 757

[48] Liao X., Brown K. A., Schmucker A. L., Liu G., He S., Shim W., Mirkin C. A. Nat. Commun., 2013, 4, 2103

[49] Carroll K. M., Giordano A. J., Wang D., Kodali V. K., Scrimgeour J., King W. P., Marder S. R., Riedo E., Curtis J. E., Langmuir, 2013, 29, 8675

[50] Carbonell C., Valles D., Wong A. M., Carlini A. S., Touve M. A., Korpanty J., Gianneschi N. C., Braunschweig A. B., Nat. Commun., 2020, 11, 1244

[51] Li Y., Maynor B. W., Liu J., J. Am. Chem. Soc., 2001, 123, 2105

[52] Sheehan P. E., Whitman L. J., King W. P., Nelson B. A., Appl. Phys. Lett. 2004, 85, 1589

[53] Huo F., Zheng G., Liao X., Giam L. R., Chai J., Chen X., Shim W., Mirkin C. A., Nat. Nanotechnol., 2010, 5, 637

[54] Huang Z., Li L., Zhang X. A., Alsharif N., Wu X., Peng Z., Cheng X., Wang P. Brown K. A., Wang Y., Adv. Mater., 2018, 30, 1705303

[55] Lee S., Cho N. P., Kim J. D., Jung H., Kang S. H., Analyst, 2009, 134, 933

[56] Lenhert S., Brinkmann F., Laue T., Walheim S., Vannahme C., Klinkhammer S., Xu M., Sekula S., Mappes T., Schimmel T., Fuchs H., Nat. Nanotechnol. 2010, 5, 275

[57] Laing S., Irvine E. J., Hernandez-Santana A., Smith W. E., Faulds K., Graham D., Anal. Chem., 2013, 85, 5617

[58] Zhou D. J., Sinniah K., Abell C., Rayment T., Angew. Chem. Int. Ed., 2003 42,4934

[59] Lee K. B., Kim E. Y., Mirkin C. A., Wolinsky S. M., Nano Lett., 2004, 4, 1869

[60] Brinkmann F., Hirtz M., Haller A., Gorges T. M., Vellekoop M. J., Riethdorf S., Muller V., Pantel K., Fuchs H., Sci. Rep., 2015, 5, 15342

[61] Salaita K., Wang Y., Fragala J., Vega R. A., Liu C., Mirkin C. A., Angew. Chem Int. Ed., 2006, 45, 7220

[62] Liu X., Li Y., Zheng Z., Nanoscale, 2010, 2, 2614
[63] Xie Z., Chen C., Zhou X., Gao T., Liu D., Miao Q., Zheng Z., ACS Appl. Mater. Interfaces, 2014, 6, 11955

[64] Lowry T. W., Prommapan P., Rainer Q., van Winkle D., Lenhert S., Sensors, 2015, 15, 2086

65] Basabe-Desmonts L., Wu C. C., van der Werf K. O., Peter M., Bennink M. Otto C., Velders A. H., Reinhoudt D. N., Subramaniam V., Crego-Calama M., ChemPhysChem, 2008, 9, 1680

[66] Martinez-Otero A., Hernando J., Ruiz-Molina D., Maspoch D., Small, 2008, 4, 2131

[67] Ma X., Li F., Xie Z., Xue M., Zheng Z., Zhang X., Soft Matter, 2017, 13, 3685 [68] Cabezas M. D., Mirkin C. A., Mrksich M., Nano Lett, 2017, 17, 1373

[69] Manandhar P., Chen K. S., Aledealat K., Mihajlovic G., Yun C. S., Field M., Sullivan G. J., Strouse G. F., Chase P. B., von Molnar S., Xiong P., Nanotechnology, 2009, 20, 355501

[70] Lee S., Lee S., Ko Y. H., Jung H., Kim J. D., Song J. M., Choo J., Eo S. K., Kang S. H., Talanta, 2009, 78, 608

[71] Thompson D. G., McKenna E. O., Pitt A., Graham D., Biosens. Bioelectron., 2011, 26, 4667

[72] Petersson L., Coen M., Amro N. A., Truedsson L., Borrebaeck C. A. K., Wingren C., Bioanalysis, 2014, 6, 1175

[73] Petersson L., Dexlin-Mellby L., Bengtsson A. A., Sturfelt G., Borrebaeck C. A., Wingren C., Lab Chip, 2014, 14, 1931

[74] Mitsakakis K., Sekula-Neuner S., Lenhert S., Fuchs H., Gizeli E., Analyst, 2012, 137, 3076

[75] Hirtz M., Oikonomou A., Georgiou T., Fuchs H., Vijayaraghavan A., Nat. Commun., 2013, 4, 2591

[76] Hirtz M., Oikonomou A., Clark N., Kim Y. J., Fuchs H., Vijayaraghavan A., Nanoscale, 2016, 8, 15147

[77] Bog U., Laue T., Grossmann T., Beck T., Wienhold T., Richter B., Hirtz M., Fuchs H., Kalt H., Mappes T., Lab Chip, 2013, 13, 2701

[78] Lowry T. W., Hariri H., Prommapan P., Kusi-Appiah A., Vafai N., Bienkiewicz E. A., van Winkle D. H., Stagg S. M., Lenhert S., Small, 2016, 12, 506

[79] Li S. F., Szegedi S., Goluch E., Liu C., Anal. Chem., 2008, 80, 5899

[80] Stokes R. J., Dougan J. A., Graham D., Chem. Commun., 2008, 44, 5734

[81] Heydari E., Photonic Nanostrut., 2019, 36, 100708

[82] Rani E., Mohshim S. A., Ahmad M. Z., Goodacre R., Ahmad S. A. A., Wong L. S., Polymers, 2019, 11, 561

[83] Ruemmele J. A., Hall W. P., Ruvuna L. K., van Duyne R. P., Anal. Chem., 2013, 85, 4560

[84] Sekula-Neuner S., Maier J., Oppong E., Cato A. C., Hirtz M., Fuchs H., Small, 2012, 8, 585

[85] Oppong E., Hedde P. N., Sekula-Neuner S., Yang L., Brinkmann F., Dorlich R. M., Hirtz M., Fuchs H., Nienhaus G. U., Cato A. C., Small, 2014, 10, 1991

[86] Kumar R., Bonicelli A., Sekula-Neuner S., Cato A. C., Hirtz M., Fuchs H. Small, 2016, 12, 5330

[87] Liu H., Liu X., Meng J., Zhang P., Yang G., Su B., Sun K., Chen L., Han D., Wang S., Jiang L., Adv. Mater., 2013, 25, 922

[88] Zhang P., Chen L., Xu T., Liu H., Liu X., Meng J., Yang G., Jiang L., Wang S., Adv. Mater., 2013, 25, 3566

[89] Li Y., Lu Q., Liu H., Wang J., Zhang P., Liang H., Jiang L., Wang S., Adv. Mater., 2015, 27, 6848

[90] Meng J., Zhang P., Zhang F., Liu H., Fan J., Liu X., Yang G., Jiang L., Wang S., ACS Nano, 2015, 9, 9284

[91] Wang S., Liu K., Liu J., Yu Z. T. F., Xu X., Zhao L., Lee T., Lee E. K., Reiss J., Lee Y.-K., Chung L. W. K., Huang J., Rettig M., Seligson D., Duraiswamy K. N., Shen C. K. F., Tseng H.-R., Angew. Chem. Int. Ed., 2011, 50, 3084

[92] Wang S., Wang H., Jiao J., Chen K.-J., Owens G. E., Kamei K.-I., Sun J., Sherman D. J., Behrenbruch C. P., Wu H., Tseng H.-R., Angew. Chem. Int. Ed., 2009, 48, 8970

[93] Liu H. Y., Koch C., Haller A., Joosse S. A., Kumar R., Vellekoop M. J., Horst L. J., Keller L., Babayan A., Failla A. V., Jensen J., Peine S., Keplinger F., Fuchs H., Pantel K., Hirtz M., Adv. Biosyst., 2020, 4, 1900162

[94] Brinkmann F., Hirtz M., Greiner A. M., Weschenfelder M., Waterkotte B., Bastmeyer M., Fuchs H., Small, 2013, 9, 3266

[95] Carbonell C., Valles D. J., Wong A. M., Tsui M. W., Niang M., Braunschweig A. B. Chem, 2018, 4, 857

[96] Zheng Z., Daniel W. L., Giam L. R., Huo F., Senesi A. J., Zheng G., Mirkin C. A., Angew. Chem. Int. Ed., 2009, 48, 7626

[97] Kumar R., Weigel S., Meyer R., Niemeyer C. M., Fuchs H., Hirtz M., Chem. Commun., 2016, 52, 12310

[98] Radha B., Liu G., Eichelsdoerfer D. J., Kulkarni G. U., Mirkin C. A., ACS Nano, 2013, 7, 2602

[99] Zhao J., Swartz L. A., Lin W.-F., Schlenoff P. S., Frommer J., Schlenoff J. B., Liu G.-Y., ACS Nano, 2016, 10, 5656

[100] Xiong E., Jiang L., Tian T., Hu M., Yue H., Huang M., Lin W., Jiang Y., Zhu D., Zhou X., Angew. Chem. Int. Ed., 2021, 60, 5307 